NISSUNA UMANA INVESTIGAZIONE SI PUO DIMANDARE VERA SCIENZIA S'ESSA NON PASSA PER LE MATEMATICHE DIMOSTRAZIONI LEONARDO DA VINCI

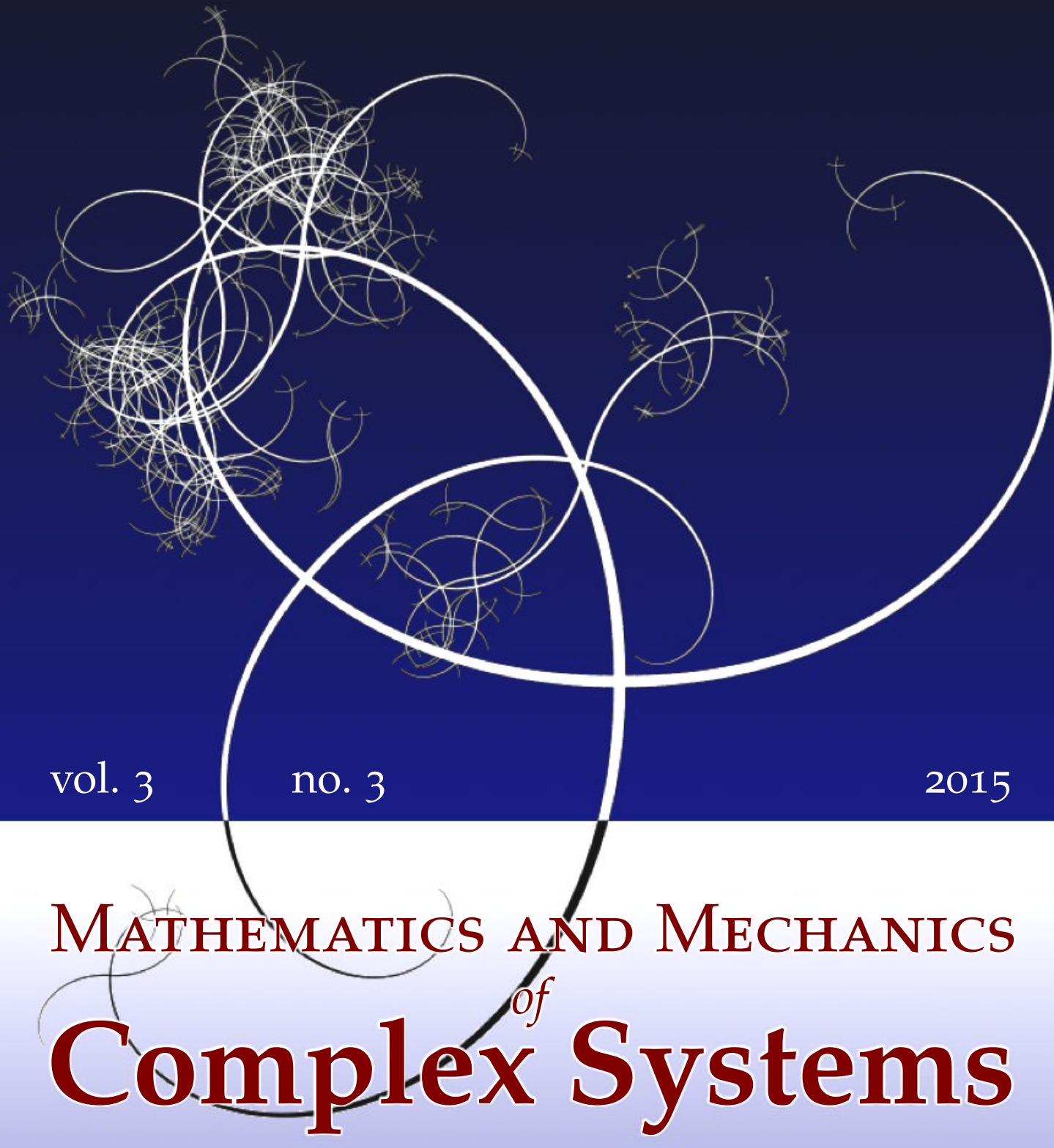

Anil Misra And PAyAm PoORsolhjouy

IDENTIFICATION OF HIGHER-ORDER ELASTIC CONSTANTS FOR GRAIN ASSEMBLIES BASED UPON GRANULAR MICROMECHANICS 


\title{
IDENTIFICATION OF HIGHER-ORDER ELASTIC CONSTANTS FOR GRAIN ASSEMBLIES BASED UPON GRANULAR MICROMECHANICS
}

\author{
ANil Misra And PAYAm PoOrsolhjouy
}

\begin{abstract}
Macroscale behavior of granular media is characterized by the significant effects of grain-pair interactions and the microstructure of each grain neighborhood. From a continuum viewpoint, granular materials may be modeled as micromorphic media to account for their complex grain-scale (microscale) kinematics. To this end we express the grain displacement in terms of the neighboring grain displacements utilizing the Taylor series expansion. The introduced gradients in the Taylor series are identified in terms of the macroscale deformation measures introduced in microstructural elasticity and micromorphic mechanics. As a result, a continuum model of the granular media is derived enriched by nonclassical terms, including terms that model grain displacement fluctuations and higher gradients of displacements. In the derived model, the continuum stiffness tensors are obtained in terms of grain-pair stiffness coefficients and fabric parameters defining the geometry of grains and their contacts. To identify the elastic constants of the enhanced continuum model, we perform numerical experiments on grain assemblies using discrete simulations subjected to relevant boundary conditions. The need for additional macroscale deformation measures for the continuum modeling of granular materials becomes evident in this identification process. The obtained elastic constants are then used to determine the microscale (or grain-pair) stiffness coefficients applicable to the continuum model. These grain-scale stiffness coefficients are found to be affected by the heterogeneity of microstructure.
\end{abstract}

\section{Introduction}

Among the various forms of granular materials, the grain assemblies formed of grains in noncohesive contacts more strongly exhibit the microstructural and micromechanical effects at the macroscale behavior. For a macroscale description of these materials, therefore, approaches are needed that can readily represent

\section{Communicated by Angela Madeo.}

Research supported in part by the United States National Science Foundation grant CMMI-1068528. MSC2010: 74Q15.

Keywords: granular micromechanics, micromorphic continuum, micro-macro identification, discrete element. 


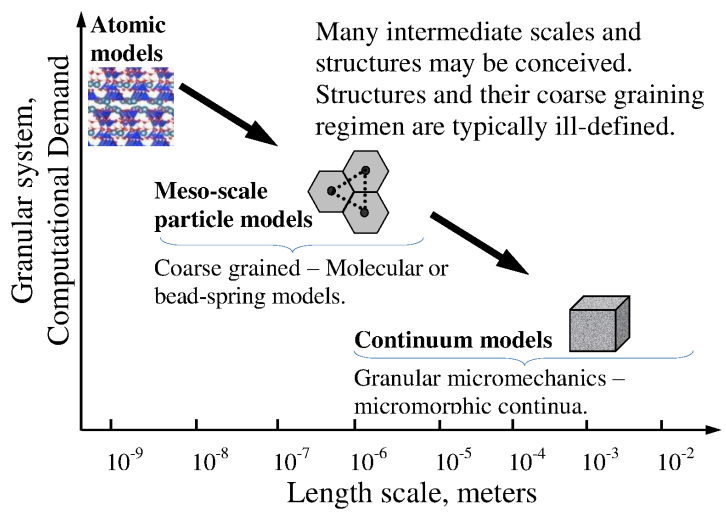

Figure 1. Schematic representation of modeling length scales and the corresponding computational demand.

the effects of grain-pair interactions. Discrete models based upon various coarsegraining schemes as schematically depicted in Figure 1 are not desirable for many problems in science and engineering that deal with structures that may contain large numbers of grains $\left(>10^{6}\right)$. These models are not only computationally expensive, but require local constitutive laws for grain-pair interactions and simulated microstructures, which are often not readily conceivable. Multiscale models that seek a hybrid discrete-continuum approach also suffer from reliance on information at various scales that is not readily available or easily conceived for complex material systems. Nonclassical continuum models are needed that can capture the effects of microscale mechanisms. Indeed, the necessity of modeling microscale mechanisms within the rubric of continuum mechanics is made clear in pioneering works such as [Cosserat and Cosserat 1909; Eringen 1999; Germain 1973; Green and Rivlin 1964; Mindlin 1964; Toupin 1964]. The seminal developments of continuum mechanics are known to proceed from some micromechanical conception of deformable materials, as seen from the works of Piola [Auffray et al. 2015; dell'Isola et al. 2014], Navier [1827] and Cauchy [1826-1830].

An important aspect of deformation of grain packing is nonaffine particle movement when a volume element of these materials is subjected to uniform boundary conditions. This aspect has been observed experimentally [Misra 1998; Misra and Jiang 1997], through many numerical simulations (see among others [Peters and Walizer 2013]), and in models of these materials within the classical continuum mechanics framework [Jenkins et al. 2005; Misra and Chang 1993; Trentadue 2001]. To model the effects of fluctuations in grain motions (termed as micro-deformations or inner motions) it is advantageous to enrich the classical continuum approach [Maugin 2014]. To that end we combine the granular micromechanics paradigm, which offers a robust methodology for developing continuum models of granular material systems by incorporating microscale effects [Misra and Singh 2014; 
2015; Misra and Poorsolhjouy 2015c], with the identification of grain (microscale) motions in terms of the Mindlin-Eringen macroscale deformation measures introduced in microstructural elasticity [Mindlin 1964] and micromorphic mechanics [Eringen 1999]. As a result, an enhanced continuum model of the granular media is obtained, enriched by nonclassical terms that model grain displacement fluctuations and higher gradients of displacements [Misra and Poorsolhjouy 2015b; 2015a]. In addition, expressions are derived for effective macroscopic constitutive coefficients corresponding to (1) the macroscale displacement gradient, (2) the fluctuations in displacement gradient which is related to the microscale displacement gradient, and (3) the macroscale gradient of microdisplacement gradient or the second gradient of displacement. In this paper, we demonstrate a method for identifying the elastic constants of the enhanced continuum model based upon numerical experiments on grain assemblies using discrete simulations. Two types of grain assemblies are analyzed: (1) regular closed-packed assembly of equaldiameter disks with vacancy type defects, and (2) irregular assembly of disks of three different sizes. These assemblies are treated as $2 \mathrm{D}$ granular media for modeling purposes. Discrete simulations are performed using unit cells of these grain assemblies to obtain deformation energies under prescribed displacement boundary conditions compatible with uniform continuum kinematic measures. These deformation energies are then analyzed to identify the continuum elastic constants. The need for additional macroscale deformation measures for the continuum modeling of granular materials becomes evident in this identification process. The obtained elastic constants are used to determine the microscale (or grain-pair) stiffness coefficients applicable to the continuum model. These grain-scale stiffness coefficients are found to be affected by the heterogeneity of microstructure, indicating that they do not represent stiffness of an isolated grain-pair; rather they represent a collective stiffness behavior of the grain-pair and its neighbors.

\section{Enhanced continuum model of granular media}

2.1. Micro-macro kinematic identification. We consider a unit cell representative of the granular assembly. In a continuum model, the unit cell plays the role of a material point $P$, as depicted in Figure 2, where the macroscale coordinate system $\boldsymbol{x}$ only distinguishes different material points. In addition, a coordinate system $\boldsymbol{x}^{\prime}$ is attached to the material point, $P$, at the barycenter of the unit cell with coordinate axes parallel to those of the macroscale coordinate system $\boldsymbol{x}$. Following the methodology of microstructural elasticity described by Mindlin [1964], we define the micro-deformation gradient, $\psi_{i j}\left(x_{k}\right)=\phi_{i, j}\left(x_{k}\right)$, which is decomposed into a part representing the average or macroscale displacement gradient, $\bar{\phi}_{i, j}$, and a second part, $\gamma_{i j}$, representing the gradient of the displacement fluctuations with 


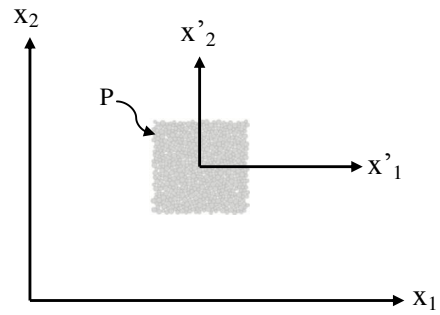

Figure 2. Representation of material point and the global coordinate system, $\boldsymbol{x}$, and the local coordinate system, $\boldsymbol{x}^{\prime}$, located at the material point's center of mass.

respect to $\boldsymbol{x}^{\prime}$, as

$$
\psi_{i j} \triangleq \phi_{i, j}=\bar{\phi}_{i, j}-\gamma_{i j}
$$

From the viewpoint of identifying constitutive relationships, we consider the continuum to be subjected to a linear macroscale displacement field. In this case, the macroscale displacement gradient, $\bar{\phi}_{i, j}$, is independent of coordinates $x_{k}$. Further, the grain displacement fluctuations are assumed to be linear functions of the local coordinate system $\boldsymbol{x}^{\prime}$, leading to a micromorphic theory of degree 1, in the terminology introduced by Germain [1973]. This implies that the second-rank tensor $\gamma_{i j}$ is independent of the local coordinates (it is homogeneous within the material point) and is a function only of $x_{k}$. Throughout the paper, the subscripts follow tensor notation and the summation convention over repeated subscripts is implied unless explicitly noted otherwise.

We now consider the relative displacement of two contacting neighbor grains, $n$ and $p$, within the unit cell of the granular media. Displacement of grain $p$ is written using a Taylor series expansion of the displacement of the neighbor grain, $n$, with terms up to second order, as

$$
\delta_{i}^{n p}=\phi_{i}^{p}-\phi_{i}^{n}=\phi_{i, j}^{n} l_{j}+\frac{1}{2} \phi_{i, j k}^{n} l_{j} l_{k}+\cdots,
$$

where $\phi_{i}$ is the displacement of grain centroids, and $l_{j}$ is the vector joining the centroids of the two grains, $n$ and $p$ (denoted by the superscripts). Using (1) and (2), the relative displacement of two neighbor grains, $n$ and $p$, is derived as

$$
\delta_{i}^{n p}=\left(\bar{\phi}_{i, j}-\gamma_{i j}\right) l_{j}+\frac{1}{2} \phi_{i, j k} l_{j} l_{k}=\bar{\phi}_{i, j} l_{j}-\gamma_{i j} l_{j}+\frac{1}{2} \phi_{i, j k} l_{j} l_{k}=\delta_{i}^{M}-\delta_{i}^{m}+\delta_{i}^{g} .
$$

As seen from (3), the intergranular relative displacements between two interacting (contacting) grains is decomposed into three terms:

(1) $\delta_{i}^{M}$, due to the macroscale displacement gradient, $\bar{\phi}_{i, j}$;

(2) $\delta_{i}^{m}$, due to the gradients of the fluctuation in grain displacement, $\gamma_{i j}(x)$; and 
(3) $\delta_{i}^{g}$, due to the second gradient term, $\phi_{i, j k}$, which is same as the gradient of the relative displacements, $\gamma_{i j, k}$.

The three relative displacement terms, denoted by the superscripts $M, m$ and $g$, are

$$
\delta_{i}^{M}=\bar{\phi}_{i, j} l_{j} ; \quad \delta_{i}^{m}=\gamma_{i j} l_{j} ; \quad \delta_{i}^{g}=\frac{1}{2} \phi_{i, j k} l_{j} l_{k}=\phi_{i, j k} J_{j k}=\psi_{i j, k} J_{j k},
$$

and the tensor $J_{i j}=l_{i} l_{j} / 2$ represents a moment tensor introduced here for simplifying further derivations.

The relative rotation of grains within the granular assembly can be related to the rotation field within the material point, defined as the curl of displacement field [Misra and Poorsolhjouy 2015b]. Thus, applying Taylor series expansion, the relative rotation of two neighbor grains, $n$ and $p$, denoted as $\boldsymbol{\theta}$, is obtained as

$$
\theta_{i}^{n p}=\kappa_{i}^{p}-\kappa_{i}^{n}=\kappa_{i, p}^{n} l_{p}=\left(e_{i j k} \phi_{k, j}\right)_{, p} l_{p}=e_{i j k} \phi_{k, j p} l_{p} .
$$

The intergranular relative rotation between two interacting grains is, thus, related to the second gradient term, $\phi_{i, j k}$. That grains undergo relative rotations is also known from measurements of kinematic fields in grain assembles [Misra 1998; Misra and Jiang 1997].

2.2. Stress and force conjugates to macro-micro kinematic measures. For further development, we retain only the symmetric part of the macroscale displacement gradient tensor, $\bar{\phi}_{(i, j)}$, as the classical small-deformation strain tensor, and express the macroscale deformation energy density of the granular continua as a function of the continuum kinematic measures as $W=W\left(\bar{\phi}_{(i, j)}, \gamma_{i j}, \phi_{i, j k}\right)$. The macroscale stress components conjugate to the kinematic measures are then defined as

$$
\tau_{i j}=\frac{\partial W}{\partial \bar{\phi}_{(i, j)}}=\frac{\partial W}{\partial \epsilon_{i j}}, \quad \sigma_{i j}=\frac{\partial W}{\partial \gamma_{i j}}, \quad \mu_{i j k}=\frac{\partial W}{\partial \gamma_{i j, k}},
$$

where $\tau_{i j}, \sigma_{i j}$, and $\mu_{i j k}$ are Cauchy stress, relative stress, and double stress, respectively. We note that the macroscopic strain energy density function can be obtained as the volume average of the deformation energies of the grain-pair interactions, written as

$$
W=\frac{1}{V} \sum_{\alpha} W^{\alpha}\left(\delta_{i}^{M}, \delta_{i}^{m}, \delta_{i}^{g}, \theta_{i}^{u}\right),
$$

where the superscript $\alpha$ denotes the $\alpha$-th grain-pair interaction, and it has been intentionally dropped from the kinematic measures to simplify the equations. The forces and moment conjugate to the microscale kinematic measures are defined as

$$
\frac{\partial W^{\alpha}}{\partial \delta_{i}^{\alpha \xi}}=f_{i}^{\alpha \xi}, \quad \text { where } \xi=M, m, g, \quad \text { and } \quad \frac{\partial W^{\alpha}}{\partial \theta_{i}^{\alpha u}}=m_{i}^{\alpha u} .
$$


Now, substituting (8) and (7) into (6) and using (4) and (5), respectively, macroscopic stress tensors conjugate to the macroscale kinematic measures are obtained as

$$
\begin{aligned}
3 \tau_{i j}=\frac{\partial W}{\partial \epsilon_{i j}} & =\frac{1}{V} \sum_{\alpha} \frac{\partial W^{\alpha}}{\partial \delta_{k}^{M}} \frac{\partial \delta_{k}^{M}}{\partial \epsilon_{i j}}=\frac{1}{V} \sum_{\alpha} f_{i}^{M \alpha} l_{j}^{\alpha}, \\
\sigma_{i j}=\frac{\partial W}{\partial \gamma_{i j}} & =\frac{1}{V} \sum_{\alpha} \frac{\partial W^{\alpha}}{\partial \delta_{k}^{m}} \frac{\partial \delta_{k}^{m}}{\partial \gamma_{i j}}=\frac{1}{V} \sum_{\alpha} f_{i}^{m \alpha} l_{j}^{\alpha}, \\
\mu_{i j k}=\frac{\partial W}{\partial \phi_{i, j k}} & =\frac{1}{V} \sum_{\alpha}\left(\frac{\partial W^{\alpha}}{\partial \delta_{l}^{g}} \frac{\partial \delta_{l}^{g}}{\partial \phi_{i, j k}}+\frac{\partial W^{\alpha}}{\partial \theta_{l}^{u}} \frac{\partial \theta_{l}^{u}}{\partial \phi_{i, j k}}\right) \\
& =\frac{1}{V}\left(\sum_{\alpha} f_{i}^{g \alpha} J_{j k}^{\alpha}+\sum_{\alpha} m_{l}^{u \alpha} e_{j i l} l_{k}^{\alpha}\right) .
\end{aligned}
$$

Thus, the stress tensor conjugates are related to the microscale force measures and moments. Similar expressions have been reported in literature [Chen and Lee 2003] based upon the generalization of the virial theorem [Ganghoffer 2010]. However, it is notable that, in the stress tensor expressions presented here, separate force measures conjugate to the grain-pair (microscale) counterparts of the continuum deformation measures are defined, which is clearly different from those based upon the virial theorem.

2.3. Microscale and macroscale constitutive equations. For linear isotropic elasticity, the following quadratic form of $W^{\alpha}$ is formulated:

$$
W^{\alpha}=\frac{1}{2}\left[\sum_{\xi} K_{i j}^{\alpha \xi} \delta_{i}^{\alpha \xi} \delta_{j}^{\alpha \xi}+G_{i j}^{\alpha u} \theta_{i}^{\alpha u} \theta_{j}^{\alpha u}\right], \quad \text { where } \xi=M, m, g,
$$

where $K$ and $G$ represent the grain-pair stiffness parameters for forces and moments, respectively. Thus, the microscale constitutive equations that link the microscale kinematics measures to their conjugate force and moment measures take the form

$$
\begin{aligned}
f_{i}^{\alpha \xi} & =K_{i j}^{\alpha \xi} \delta_{j}^{\alpha \xi}, \quad \text { where } \xi=M, m, g, \\
m_{i}^{\alpha u} & =G_{i j}^{\alpha u} \theta_{j}^{\alpha u} .
\end{aligned}
$$

The introduced grain-pair stiffness parameters define the force conjugates associated with different microscale kinematic measures that contribute to intergranular relative displacements and rotations. These stiffness parameters do not represent the stiffness of two isolated interacting grains. In the derived model we have introduced four types of intergranular stiffness parameters, namely, the average, the fluctuation, the second gradient and the rotational, distinguished by their superscripts $M, m, g$ and $u$, respectively. We note that in (12) and (13) the terms that cross-link the different microscale kinematic measures have been ignored for 
simplicity. The consequence of this assumption will be that uncoupled constitutive relationships will be obtained for the continuum model. Further, it should be noted that the microscale stiffness coefficients in (13) represent intergranular stiffness measures in a statistical sense. Since the total relative displacement between two neighbor grains was previously decomposed into different terms, representing the average and fluctuation displacement gradients and also second gradient terms, the stiffness coefficients corresponding to them will all contribute, in a statistical sense, to form the overall response of the interaction to the grains' relative displacements. Now, substituting the microscale constitutive equations, (13), into (9)-(11), and noting that the macroscale strain measures are constant throughout the material point, macroscale constitutive equations are derived as

$$
\begin{aligned}
\tau_{i j} & =\frac{1}{V} \sum_{\alpha} f_{i}^{M} l_{j}^{\alpha}=\frac{1}{V} \sum_{\alpha} K_{i k}^{M} \delta_{k}^{M} l_{j}^{\alpha}=\left(\frac{1}{V} \sum_{\alpha} K_{i k}^{M} l_{l}^{\alpha} l_{j}^{\alpha}\right) \epsilon_{k l}=C_{i j k l}^{M} \epsilon_{k l}, \\
\sigma_{i j} & =\frac{1}{V} \sum_{\alpha} f_{i}^{m} l_{j}^{\alpha}=\frac{1}{V} \sum_{\alpha} K_{i k}^{m} \delta_{k}^{\alpha} l_{j}^{\alpha}=\left(\frac{1}{V} \sum_{\alpha} K_{i k}^{m} l_{l}^{\alpha} l_{j}^{\alpha}\right) \gamma_{k l}=C_{i j k l}^{m} \gamma_{k l}, \\
\mu_{i j k} & =\frac{1}{V} \sum_{\alpha}\left(f_{i}^{g} J_{j k}^{\alpha}+m_{l}^{u} e_{j i l} l_{k}\right) \\
& =\left(\frac{1}{V} \sum_{\alpha} K_{i l}^{g} J_{m n}^{\alpha} J_{j k}^{\alpha}+\frac{1}{V} \sum_{\alpha} G_{p q}^{u} e_{m l q} e_{j i p} l_{k} l_{n}\right) \phi_{l, m n} \\
& =\left(A_{i j k l m n}^{g}+A_{i j k l m n}^{u}\right) \phi_{l, m n}=A_{i j k l m n} \phi_{l, m n} .
\end{aligned}
$$

Here it should be noted that the summations in (14)-(16) are performed over all grain-pair interactions inside the material point. The quantities inside the summation (stiffness coefficients and geometric parameters $l_{i}$ and $J_{i j}$ ) are all different for every single grain-pair interaction. It is, however, possible to conceive of average value for all these quantities for grain-pair interactions in any given orientation. Using these average values, the summation over all grain interactions can be changed to integration over all generic orientations. To this end we define $N_{p}$ as the number density of grain-pair interactions divided by the volume of the unit cell, and $\xi$ as the normalized directional density distribution function of contacts in different orientations within the unit cell. Having a properly defined $\xi$ will enable the method to model materials with different levels of anisotropy. For modeling isotropic materials or particle assemblies with random distribution of grains in different orientations, the distribution function should take a constant value independent of the direction. So, for isotropic materials (or for randomly distributed grain assemblies), the density distribution function in 2D and 3D domains will be

$$
\xi(\theta)=\frac{1}{2 \pi} \quad \Longrightarrow \quad \int_{\theta} \xi d \theta=\frac{1}{2 \pi} 2 \pi=1 \quad(\text { for } 2 \mathrm{D})
$$


and

$$
\xi(\theta, \phi)=\frac{1}{4 \pi} \quad \Longrightarrow \quad \int_{\theta} \int_{\phi} \xi(\sin \theta d \theta d \phi)=\frac{1}{4 \pi}(2 \pi)(2)=1 \quad(\text { for } 3 \mathrm{D}) .
$$

In (17a), $\theta$ is the polar angle of the 2D polar coordinate system, while in (17b), $\theta$ and $\phi$ are the polar angles measured from the vertical axis and the azimuth angle in the 3D spherical coordinate system, respectively.

\section{Identification of elastic constants using discrete simulations}

Evidently, the enhanced continuum model of granular media derived in Section 2 can be characterized by either microscale or macroscale elastic constants. To demonstrate the applicability of the derived model, we identify these constants for specific grain assemblies using discrete simulations.

3.1. Discrete simulation methodology. For any grain within the assembly, say the $n$-th grain, the variation of the total potential energy can be written as

$$
\delta W^{n}=\sum_{\alpha} \delta W^{n \alpha}\left(\delta_{i}^{n \alpha}, \theta_{i}^{n \alpha}\right)=\sum_{\alpha} f_{i}^{n \alpha} \delta \delta_{i}^{n \alpha}+\sum_{\alpha}\left(m_{i}^{n \alpha}+e_{i j k} f_{j}^{n \alpha} r_{k}^{n}\right) \delta \theta_{i}^{n \alpha},
$$

where the summation over $\alpha$ denotes summation over all grains which have interaction with the $n$-th grain. In (18), $f^{n \alpha}, m^{n \alpha}, \delta^{n \alpha}$, and $\theta^{n \alpha}$ are the grain-pair force, moment, relative displacement, and relative rotation for grains $n$ and $\alpha$, respectively, related through the grain-scale constitutive equations for an isolated grain-pair as

$$
\begin{aligned}
f_{i}^{n \alpha} & =\bar{K}_{i j}^{\alpha} \delta_{j}^{n \alpha}, \quad \text { where } \bar{K}_{i j}^{n \alpha}=\bar{K}_{n}^{\alpha} n_{i}^{\alpha} n_{j}^{\alpha}+\bar{K}_{s}^{\alpha} s_{i}^{\alpha} s_{j}^{\alpha}+\bar{K}_{n s}^{\alpha}\left(n_{i}^{\alpha} s_{j}^{\alpha}+s_{i}^{\alpha} n_{j}^{\alpha}\right), \\
m^{n \alpha} & =\bar{G}^{\alpha} \theta^{n \alpha},
\end{aligned}
$$

where the isolated grain-pair stiffness coefficients for 2D contact of disks in the normal, tangential, and normal-tangential coupling are taken to be $\bar{K}_{n}, \bar{K}_{s}$, and $\bar{K}_{n s}$, respectively, and the grain-pair moment stiffness is taken to be $\bar{G}$.

Denoting the total force exerted on the $n$-th grain by $F_{i}$ and the total moment by $M_{i}$, the variation of the total potential energy is written as

$$
\delta W^{n}=F_{i}^{n} \delta \phi_{i}^{n}+M_{i}^{n} \delta \kappa_{i}^{n} .
$$

Further, the variation of the relative kinematic measures can be derived based on the variation of displacement and rotation fields as

$$
\begin{aligned}
& \delta \delta_{i}^{n \alpha}=\delta\left(\phi_{i}^{\alpha}-\phi_{i}^{n}\right)=-\delta \phi_{i}^{n}, \\
& \delta \theta_{i}^{n \alpha}=\delta\left(\kappa_{i}^{\alpha}-\kappa_{i}^{n}\right)=-\delta \kappa_{i}^{n} .
\end{aligned}
$$


Setting (18) and (20) equal and substituting (21), the following balance equations for grain, $n$, is found:

$$
\begin{aligned}
F_{i}^{n} & =-\sum_{\alpha} f_{i}^{n \alpha}, \\
M_{i}^{n} & =-\sum_{\alpha}\left(m_{i}^{n \alpha}+e_{i j k} f_{j}^{n \alpha} r_{k}^{n}\right) .
\end{aligned}
$$

Combining (19) and (22), the total grain force and moment can be related to the grain motions. These expressions can be assembled to form an overall forcedisplacement equation governing the behavior of the assembly as a whole, written as [Chang and Misra 1989]

$$
\{F\}_{3 M \times 1}=[S]_{3 M \times 3 M}\{u\}_{3 M \times 1},
$$

where $M$ denotes the total number of grains within the whole assembly. For each grain there are three kinematic measures (two displacements and one rotation), resulting in a total number of $3 M$ kinematic variables represented in $u$. Also, each grain has three force measures (two forces and one moment), resulting in a total number of $3 M$ force variables represented in $F$. So the overall number of variables in (23) is $6 M$, knowing any $3 M$ of which the other $3 M$ can be derived by solving the system of equations represented in matrix form in (23).

3.2. Methodology for identification of constitutive coefficients. Using (6), the macroscale strain energy can be written as

$$
W=C_{i j k l}^{M} \epsilon_{i j} \epsilon_{k l}+C_{i j k l}^{m} \gamma_{i j} \gamma_{k l}+A_{i j k l m n} \psi_{i j, k} \psi_{l m, n} .
$$

For finding the components of the stiffness tensors, numerical experiments are performed with $2 \mathrm{D}$ grain assembly using the discrete simulation method. These numerical experiments are performed in a manner akin to physical experiments by applying boundary conditions on the grain assemblies. Thus, to obtain the components of the stiffness tensor, $C_{i j k l}^{M}$, we purely apply on the boundary of the grain assembly a specified macroscale displacement gradient while constraining the fluctuation displacement gradient, $\gamma_{i j}$, and the second gradient term, $\psi_{i j, k}$, to be zero. In order to achieve this type of displacement at the boundary, boundary grains and their immediate neighbors are displaced by the amount compatible with the specified macroscale displacement gradient. In this manner, the fluctuations in the displacement gradient and the second gradient of displacement are ensured to be zero. For the assembly loaded in this manner, the strain energy will be

$$
\left.W\right|_{\left(\gamma_{i j}=0, \psi_{i j, k}=0\right)}=C_{i j k l}^{M} \epsilon_{k l} \epsilon_{i j} .
$$


Thus, by performing a sufficient number of simulations with specified combinations of the strain components, the elastic constants are identified.

For finding the components of $C_{i j k l}^{m}$, we purely apply fluctuations in displacement gradient while keeping the macroscale displacement gradient and the second gradient of displacement to be zero. For this type of boundary condition, the boundary grains are kept stationary while their immediate neighbors are moved according to a fluctuation in displacement gradient using (3). By loading the assembly in this manner, the strain energy of the assembly will be

$$
\left.W\right|_{\left(\epsilon_{i j}=0, \psi_{i j, k}=0\right)}=C_{i j k l}^{m} \gamma_{i j} \gamma_{k l} .
$$

Applying a sufficient number of combinations of fluctuation strain components as for the case of macrostrain, the components of the tensor $C_{i j k l}^{m}$ are derived. Finally, for finding the components of the sixth-rank stiffness tensors, second gradients of displacement fluctuations are applied on the assembly while keeping the average and fluctuations in displacement gradient to be equal to zero. For this purpose the boundary grains are kept stationary while their immediate neighbors are moved in consistence with the second gradient in the displacement fluctuations. Movements of the neighbors of boundary grains are derived using (3) with the desired value of $\psi_{i j, k}$. In this loading scheme the internal strain energy will be derived as

$$
\left.W\right|_{\left(\epsilon_{i j}=0, \gamma_{i j}=0\right)}=A_{i j k l m n} \psi_{i j, k} \psi_{l m, n} .
$$

For a $2 \mathrm{D}$ granular system, the sixth-rank stiffness tensor $A_{i j k l m n}$ can be written as an $8 \times 8$ matrix with 36 independent components. To find all these components, second gradient of displacement in 36 different combinations need to be applied to the material. Eight of these combinations are indicated by the eight different components of $\psi_{i j k}$ and the 26 remaining ones are composed of their combinations. Finding the energy for each one of these cases, using (18) and writing (27), result in a system of 36 equations and 36 unknowns that, when solved, will yield all components of the second gradient stiffness tensor.

\section{Results}

Two types of grain assemblies are analyzed: (1) regular closed-packed assembly of equal-diameter disks (monodisperse) with vacancy type defects, and (2) irregular assembly of three different diameter disks (tridisperse). These assemblies are treated as 2D granular media for modeling purposes.

4.1. Regular monodispersed grain assembly. Regular hexagonal closed-packed assemblies from cylindrical particles (assembly A, seen in Figure 3, left) have been analyzed. Further, defected assemblies are also made by removing grains from the assembly once every four rows (assembly B, seen in Figure 3, middle) and once 

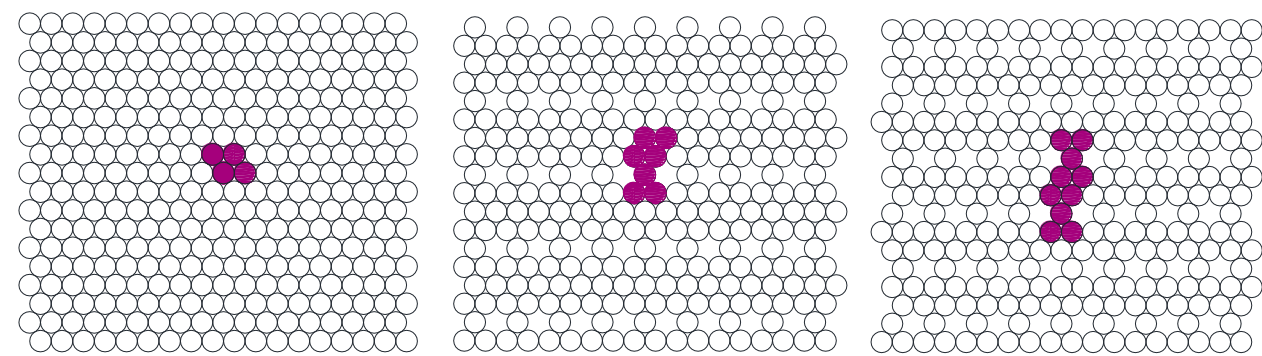

Figure 3. Three regular assemblies. Left: hexagonal closed-pack (assembly A). Middle and right: defected assemblies (assemblies $\mathrm{B}$ and $\mathrm{C}$, respectively).

every three rows (assembly $\mathrm{C}$, seen in Figure 3, right). Thus, Assembly A is the densest of the three, $\mathrm{B}$ is intermediate, and $\mathrm{C}$ is the loosest.

Assembly A has planes of elastic symmetry every 30 degrees. Applying the effects of elastic symmetries about horizontal and vertical planes shows that the material behaves as an orthotropic material in 2D with four independent constants in its first gradient stiffness tensor. Considering elastic symmetry about the plane whose normal vector makes a $150^{\circ}$ angle with the positive $x$-axis results in a stiffness tensor with only two independent constants, as is the case for isotropic materials. Defected assemblies (B and C) are also isotropic because the defects are chosen so that the removed contacts will not disrupt the symmetry in intergranular forces.

For each assembly, a unit cell is defined. By periodically repeating the unit cell in both horizontal and vertical directions, the complete assembly is formed. The unit cells can be seen in Figure 3 by shaded grains. Discrete simulations of these unit cells with periodic boundary conditions have been performed. In these simulations, intergranular stiffness coefficients are assigned as $\bar{K}_{n}=2 \bar{K}_{s}=17.5 \mathrm{KN} / \mathrm{mm}$ and $\bar{K}_{n s}=\bar{G}=0.0$. For the three assemblies, multiple simulations, as discussed in Section 3.2, have been performed, and stiffness tensors corresponding to average displacement gradient, fluctuations in displacement gradient, and second gradients are derived and given in Table 1. For assembly A, since all grains in the unit cell are in fact boundary grains, stiffness tensors corresponding to displacement fluctuations and second gradient terms are zero. Indeed, in such grain assembly, all grains always move according to the average displacement gradient applied on the boundary grains. For the two other assemblies, however, not all grains inside the unit cell are boundary grains. Thus, effects of grain displacement fluctuations and second gradient of displacement will be nonzero. Components of the fourth-rank stiffness tensors, $\boldsymbol{C}^{M}$ and $\boldsymbol{C}^{m}$, and the sixth-rank stiffness tensor corresponding to second gradient terms, $\boldsymbol{A}=\boldsymbol{A}^{u}+\boldsymbol{A}^{g}$, for these regular assemblies are presented in Table 1. It is noteworthy that the stiffness components associated with fluctuation and second gradient increase as more defects are introduced into the assembly. 


\begin{tabular}{|c|c|c|c|c|c|c|}
\hline \multicolumn{4}{|c|}{$C_{i j k l}^{M}$ and $C_{i j k l}^{m}$} & \multicolumn{3}{|c|}{$A_{i j k l m n}$} \\
\hline Component & $\begin{array}{c}\text { Assembly } \\
\text { A }\end{array}$ & $\begin{array}{c}\text { Assembly } \\
\text { B }\end{array}$ & $\begin{array}{c}\text { Assembly } \\
\text { C }\end{array}$ & Component & $\begin{array}{c}\text { Assembly } \\
\text { B }\end{array}$ & $\begin{array}{c}\text { Assembly } \\
\text { C }\end{array}$ \\
\hline$C_{11}^{M}$ & 26.5 & 19.9 & 17.7 & $A_{111111}$ & 8.2 & 60.4 \\
\hline$C_{22}^{M}$ & 26.5 & 19.9 & 17.7 & $A_{112112}$ & 24.7 & 14.6 \\
\hline$C_{33}^{M}$ & 11.4 & 8.5 & 7.6 & $A_{122122}$ & 74.0 & 43.9 \\
\hline$C_{12}^{M}$ & 3.8 & 2.8 & 2.5 & $A_{211211}$ & 11.5 & 56.5 \\
\hline$C_{13}^{M}$ & 0 & 0.0 & 0.0 & $A_{212212}$ & 34.5 & 20.5 \\
\hline$C_{23}^{M}$ & 0 & 0.0 & 0.0 & $A_{222222}$ & 103.6 & 61.4 \\
\hline$C_{11}^{m}$ & 0 & 1.6 & 7.6 & $A_{111112}$ & 14.2 & 4.2 \\
\hline$C_{22}^{m}$ & 0 & 6.6 & 8.8 & $A_{111122}$ & 24.7 & 7.3 \\
\hline$C_{33}^{m}$ & 0 & 4.7 & 6.3 & $A_{111211}$ & 0.0 & 1.7 \\
\hline$C_{44}^{m}$ & 0 & 2.2 & 7.6 & $A_{111212}=A_{112211}$ & 0.0 & -1.5 \\
\hline$C_{12}^{m}$ & 0 & 0.0 & -0.3 & $A_{111222}=A_{122211}$ & 0.0 & -2.5 \\
\hline$C_{13}^{m}$ & 0 & 2.7 & 2.7 & $A_{112122}$ & 42.7 & 25.3 \\
\hline$C_{14}^{m}$ & 0 & 0.0 & 0.0 & $A_{211212}$ & 19.9 & 5.9 \\
\hline$C_{23}^{m}$ & 0 & 0.0 & 0.0 & $A_{211222}$ & 34.5 & 10.2 \\
\hline$C_{24}^{m}$ & 0 & 3.8 & 3.8 & $A_{212222}=A_{221222}$ & 59.8 & 59.8 \\
\hline$C_{34}^{m}$ & 0 & 0.0 & -0.3 & otherwise & 0.0 & 0.0 \\
\hline
\end{tabular}

Table 1. Components of two fourth-rank stiffness tensors $\left(\boldsymbol{C}^{M}\right.$ and $\left.\boldsymbol{C}^{m}\right)$, in units of GPa, and the sixth-rank second gradient stiffness tensor $\left(\boldsymbol{A}=\boldsymbol{A}^{g}+\boldsymbol{A}^{u}\right)$, in units of N, for the regular unit cells.

4.2. Irregular tridisperse grain assembly. Three random assemblies of grains of three different sizes have been made and analyzed. The three assemblies are composed of 288, 576, and 1152 grains, as shown in Figure 4. In all three assemblies, $52 \%$ of grains have a radius of $19 \mu \mathrm{m}, 26 \%$ have a radius of $22 \mu \mathrm{m}$, and $22 \%$ have
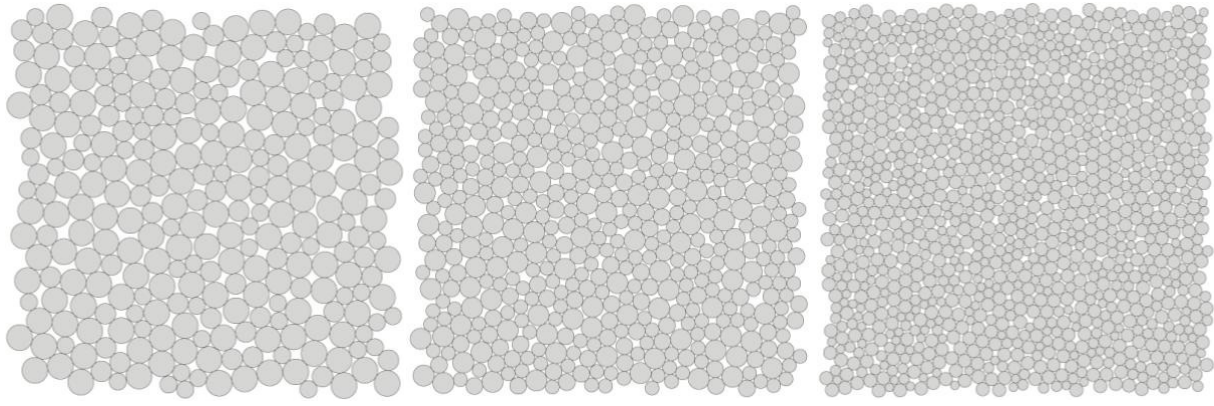

Figure 4. Irregular grain assemblies with different numbers of grains, with constant ratios of grains with different sizes. 


\begin{tabular}{|c|c|c|c|c|c|c|c|c|c|}
\hline & \multicolumn{3}{|c|}{$N=288$} & \multicolumn{3}{|c|}{$N=576$} & \multicolumn{3}{|c|}{$N=1152$} \\
\hline component & $\begin{array}{l}\text { unit } \\
\text { cell }\end{array}$ & RVE & $\begin{array}{l}\text { model } \\
\text { results }\end{array}$ & $\begin{array}{l}\text { unit } \\
\text { cell }\end{array}$ & RVE & $\begin{array}{l}\text { model } \\
\text { results }\end{array}$ & $\begin{array}{l}\text { unit } \\
\text { cell }\end{array}$ & RVE & $\begin{array}{l}\text { model } \\
\text { results }\end{array}$ \\
\hline$C_{11}^{M}$ & 17.9 & 17.7 & 17.7 & 18.9 & 19.0 & 19.0 & 18.1 & 18.3 & 18.3 \\
\hline$C_{22}^{M}$ & 17.8 & 17.7 & 17.7 & 19.0 & 19.0 & 19.0 & 18.4 & 18.3 & 18.3 \\
\hline$C_{33}^{M}$ & 7.4 & 7.4 & 7.4 & 7.9 & 7.9 & 7.9 & 7.6 & 7.6 & 7.6 \\
\hline$C_{12}^{M}$ & 2.8 & 2.8 & 2.8 & 3.1 & 3.1 & 3.1 & 3.1 & 3.0 & 3.0 \\
\hline$C_{13}^{M}$ & 0.3 & 0.0 & 0.0 & 0.2 & 0.0 & 0.0 & 0.0 & 0.0 & 0.0 \\
\hline$C_{23}^{M}$ & 0.0 & 0.0 & 0.0 & 0.1 & 0.0 & 0.0 & -0.1 & 0.0 & 0.0 \\
\hline$C_{11}^{m}$ & 4.0 & 4.2 & 4.2 & 3.1 & 2.9 & 2.9 & 2.0 & 2.0 & 2.0 \\
\hline$C_{22}^{m}$ & 4.5 & 4.2 & 4.2 & 2.8 & 2.9 & 2.9 & 2.0 & 2.0 & 2.0 \\
\hline$C_{33}^{m}$ & 3.8 & 3.7 & 3.7 & 2.4 & 2.6 & 2.6 & 1.7 & 1.8 & 1.8 \\
\hline$C_{44}^{m}$ & 3.5 & 3.7 & 3.7 & 2.7 & 2.6 & 2.6 & 1.7 & 1.8 & 1.8 \\
\hline$C_{12}^{m}$ & 0.2 & 0.2 & 2.8 & 0.1 & 0.2 & 3.1 & 0.1 & 0.1 & 3.0 \\
\hline$C_{13}^{m}$ & -0.2 & 0.0 & 0.0 & 0.1 & 0.0 & 0.0 & 0.0 & 0.0 & 0.0 \\
\hline$C_{14}^{m}$ & -0.1 & 0.0 & 0.0 & 0.0 & 0.0 & 0.0 & 0.0 & 0.0 & 0.0 \\
\hline$C_{23}^{m}$ & 0.0 & 0.0 & 0.0 & 0.0 & 0.0 & 0.0 & 0.0 & 0.0 & 0.0 \\
\hline$C_{24}^{m}$ & -0.2 & 0.0 & 0.0 & 0.0 & 0.0 & 0.0 & 0.0 & 0.0 & 0.0 \\
\hline$C_{34}^{m}$ & 0.2 & 0.2 & 0.2 & 0.1 & 0.1 & 0.1 & 0.1 & 0.1 & 0.1 \\
\hline
\end{tabular}

Table 2. Components of two fourth-rank stiffness tensors $\left(\boldsymbol{C}^{M}\right.$ and $\boldsymbol{C}^{m}$ ) for the irregular grain assemblies. All results have units of GPa. The unit cells are as given in Figure 4, the RVE is taken to be composed of randomly oriented unit cells, as in isotropic polycrystals (note the unit cells are amorphous with periodic boundaries), and model results pertain to the RVE.

a radius of $28 \mu \mathrm{m}$. Intergranular stiffness coefficients used for discrete simulations are $\bar{K}_{n}=2 \bar{K}_{s}=17.5 \mathrm{KN} / \mathrm{mm}$ and $\bar{K}_{n s}=\bar{G}=0.0$.

In Tables 2 and 3, the stiffness tensor components for the three assemblies with different numbers of grains are presented. For each assembly, first the components of the unit cell's stiffness tensor are given in the column titled "unit cell". Further, to identify the grain-pair stiffness relevant to the isotropic enhanced continuum model, we consider an RVE of granular media which comprises randomly oriented unit cells, as in polycrystals. The isotropic stiffness tensor of this RVE is estimated through the Voigt-Reuss-Hill (VRH) directional averaging process [Hill 1952]. For each assembly, in the second column in Tables 2 and 3, the components of stiffness tensors of the resulting RVE are presented. It is noteworthy that the "unit cell" results and the estimated RVEs have small differences. Finally, for each assembly, in the last columns, titled "Model results", we give the stiffness tensors 


\begin{tabular}{|c|c|c|c|c|c|c|c|c|c|}
\hline \multirow[b]{2}{*}{ component } & \multicolumn{3}{|c|}{$N=288$} & \multicolumn{3}{|c|}{$N=576$} & \multicolumn{3}{|c|}{$N=1152$} \\
\hline & $\begin{array}{l}\text { unit } \\
\text { cell }\end{array}$ & RVE & $\begin{array}{l}\text { model } \\
\text { results }\end{array}$ & $\begin{array}{l}\text { unit } \\
\text { cell }\end{array}$ & RVE & $\begin{array}{l}\text { model } \\
\text { results }\end{array}$ & $\begin{array}{l}\text { unit } \\
\text { cell }\end{array}$ & RVE & $\begin{array}{l}\text { model } \\
\text { results }\end{array}$ \\
\hline$A_{111111}$ & 206.9 & 257.9 & 257.9 & 97.1 & 100.2 & 100.2 & 33.3 & 33.9 & 33.9 \\
\hline$A_{112112}$ & 146.6 & 130.5 & 81.8 & 53.6 & 48.2 & 31.7 & 19.3 & 16.9 & 10.6 \\
\hline$A_{122122}$ & 247.8 & 233.1 & 233.1 & 82.7 & 90.3 & 90.3 & 25.7 & 29.9 & 29.9 \\
\hline$A_{211211}$ & 182.4 & 233.1 & 233.1 & 84.9 & 90.3 & 90.3 & 28.1 & 29.9 & 29.9 \\
\hline$A_{212212}$ & 143.2 & 130.5 & 81.8 & 52.6 & 48.2 & 31.7 & 19.5 & 16.9 & 10.6 \\
\hline$A_{222222}$ & 287.3 & 257.9 & 257.9 & 96.8 & 100.2 & 100.2 & 30.6 & 33.9 & 33.9 \\
\hline$A_{111112}$ & -19.4 & 0.0 & 0.0 & -3.7 & 0.5 & 0.5 & -1.2 & -0.1 & -0.1 \\
\hline$A_{111122}$ & 0.0 & -15.4 & 81.8 & 3.0 & -1.2 & 31.7 & 0.3 & -1.9 & 10.6 \\
\hline$A_{111211}$ & -3.3 & 0.0 & 0.0 & -0.9 & -0.9 & -0.9 & 0.1 & 0.1 & 0.1 \\
\hline$A_{111212}$ & 4.4 & 6.5 & 6.2 & 2.1 & 2.7 & 2.5 & 0.7 & 1.1 & 1.0 \\
\hline$A_{111222}$ & -0.8 & 0.0 & 0.0 & 0.8 & 0.0 & 0.0 & 0.2 & 0.0 & 0.0 \\
\hline$A_{112122}$ & -4.1 & 0.0 & 0.0 & 6.4 & 0.5 & 0.5 & 1.4 & -0.1 & -0.1 \\
\hline$A_{112211}$ & 3.6 & 5.9 & 6.2 & 1.8 & 2.2 & 2.5 & 0.7 & 0.9 & 1.0 \\
\hline$A_{112212}$ & -2.5 & 0.0 & 0.0 & -0.4 & 0.0 & 0.0 & 0.0 & 0.0 & 0.0 \\
\hline$A_{112222}$ & 4.9 & 6.5 & 6.2 & 1.7 & 2.7 & 2.5 & 0.8 & 1.1 & 1.0 \\
\hline$A_{122211}$ & 0.1 & 0.0 & 0.0 & 0.8 & 0.0 & 0.0 & 0.2 & 0.0 & 0.0 \\
\hline$A_{122212}$ & 4.6 & 5.9 & 6.2 & 1.0 & 2.2 & 2.5 & 0.7 & 0.9 & 1.0 \\
\hline$A_{122222}$ & 1.0 & 0.0 & 0.0 & 0.6 & 0.9 & 0.9 & -0.4 & -0.1 & -0.1 \\
\hline$A_{211212}$ & -22.8 & 0.0 & 0.0 & -4.8 & -0.5 & -0.5 & -1.2 & 0.1 & 0.1 \\
\hline$A_{211222}$ & -1.9 & -15.4 & 81.8 & 4.2 & -1.2 & 31.7 & 0.8 & -1.9 & 10.6 \\
\hline$A_{212222}$ & 3.7 & 0.0 & 0.0 & 5.4 & -0.5 & -0.5 & 1.4 & 0.1 & 0.1 \\
\hline$A_{221222}$ & 3.7 & 0.0 & 0.0 & 5.4 & -0.5 & -0.5 & 1.4 & 0.1 & 0.1 \\
\hline
\end{tabular}

Table 3. Components of the sixth-rank second gradient stiffness tensor $\left(\boldsymbol{A}=\boldsymbol{A}^{g}+\boldsymbol{A}^{u}\right)$ for the irregular grain assemblies. All results have units of $10^{-5} \mathrm{KN}$. The unit cells are as given in Figure 4, the $\mathrm{RVE}$ is taken to be composed of randomly oriented unit cells as in isotropic polycrystals (note the unit cells are amorphous with periodic boundaries), and model results pertain to the RVE.

derived from calculated microscale stiffness (i.e., grain-pair stiffness coefficients corresponding to macroscale displacement gradient, fluctuations in displacement gradients, and second gradients of displacement fluctuations). The stiffness tensors for the macroscale displacement gradients and the fluctuations in displacement gradient show a very good agreement. For the second gradient stiffness tensor, the microscale stiffness constants are found by an optimization procedure since the number of independent constants is less than the number of independent components 
in the second gradient stiffness tensors. A reasonable agreement is also found for the second gradient constitutive coefficients, and, notably, the orders of the major nonzero terms are in concurrence. We further note the need for nonzero coupling terms, $K_{n s}$, in the microscale constitutive relationships for replicating the fluctuation and second gradient macroscopic stiffness tensors. Though the effects of these stiffness components are of smaller order than those of the diagonal stiffness coefficients, $K_{n}$ and $K_{s}$, nevertheless they conform to the relationships derived from the present model (see Appendix I). We also note that the microscale moment stiffness coefficients, $G$, are negligible (of the order $10^{-16} \mathrm{~N} . \mathrm{mm}$ ), which implies that for the particular simulations the grain rotations have an insignificant role. However, this is likely a result of assumed zero rotational stiffness in the discrete simulations.

4.3. Microscale constants for enhanced continuum model. Finally, we compare the back-calculated microscale constitutive coefficients in Table 4 with those assigned in the discrete simulations. For the case of regular assembly, we observe that the microscale stiffness constants, $K_{n}^{M}$ and $K_{s}^{M}$, relevant to the macrostrain stiffness tensor for assembly A are 13\% smaller than those used in discrete simulations. The grain-pair stiffness relevant to the continuum model is clearly influenced by the strongly discrete hexagonal microstructure of the grain assembly. As the regular assembly becomes more defective (B and C), the constants, $K_{n}^{M}$ and $K_{s}^{M}$, become smaller. However, notably, the ratio $K_{s}^{M} / K_{n}^{M}$ remains 2 , which is the same as that specified in discrete simulations, thus preserving the Poisson's effect. Moreover, the microscale stiffness constants corresponding to the fluctuation and second gradient stiffness tensors appear and increase as more defects are introduced. The ratio $K_{s}^{m} / K_{n}^{m}$ no longer remains the same as that specified in discrete simulations, implying a different Poisson's effect associated with the fluctuation behavior. In addition, a coupling of the normal and shear behavior is also revealed. It is clear that the needed microscale constitutive coefficients are significantly affected by heterogeneity introduced by microstructure, so that the continuum modeling with

\begin{tabular}{|crrrrcrcc|}
\hline Assembly & \multicolumn{1}{c}{$K_{n}^{M}$} & $K_{s}^{M}$ & $K_{n}^{m}$ & $K_{s}^{m}$ & $K_{n s}^{m}$ & $K_{n}^{g}$ & $K_{s}^{g}$ & \multicolumn{1}{c|}{$K_{n s}^{g}$} \\
A & 15.2 & 7.6 & & & & & & \\
B & 10.9 & 5.4 & 2.0 & 1.7 & $2.6 \times 10^{-1}$ & 0.3 & 0.3 & $4.1 \times 10^{-2}$ \\
C & 8.4 & 4.2 & 3.3 & 3.0 & $2.3 \times 10^{-1}$ & 0.4 & 0.4 & $-3.6 \times 10^{-2}$ \\
$N=288$ & 16.8 & 7.5 & 3.6 & 2.8 & $-4.9 \times 10^{-3}$ & 0.8 & 0.7 & $1.2 \times 10^{-4}$ \\
$N=576$ & 17.1 & 7.5 & 2.3 & 1.9 & $-2.6 \times 10^{-2}$ & 0.6 & 0.5 & $-7.7 \times 10^{-3}$ \\
$N=1152$ & 16.8 & 7.2 & 1.7 & 1.3 & $1.1 \times 10^{-2}$ & 0.4 & 0.3 & $2.5 \times 10^{-3}$ \\
\hline
\end{tabular}

Table 4. Microscale constitutive coefficients derived for both the regular and irregular assemblies with $\bar{K}_{n}=2 \bar{K}_{s}=17.5 \mathrm{KN} / \mathrm{mm}$ and $\bar{K}_{n s}=\bar{G}=0.0(K$ in $\mathrm{kN} / \mathrm{mm}$ and $G$ in N.mm $)$. 
only macroscale displacement gradients is not sufficient even for the relatively uniform system analyzed here, wherein all grain pairs are given the same stiffness constants. Similar observations can be made from the comparison of microscale stiffness constants of the three irregular assemblies in which smaller particle numbers imply greater microstructural heterogeneity. Clearly, the grain neighborhoods affect the behavior of grain interactions significantly, so that the effective grainpair stiffness cannot be just estimated from the stiffness of two isolated grains. Additional macroscale deformation measures are, therefore, necessary for the continuum modeling of granular materials. Similar observations have been made for other material systems such as pantographic trusses [Alibert et al. 2003; Seppecher et al. 2011], biomaterials [Andreaus et al. 2012; 2015a], and in fiber composites [Ferretti et al. 2014]. Further, we note that the application of isolated grain-pair potentials or stiffness functions for estimating the energies and stresses associated with different deformation measures, as proposed in some multiscale models that aim to bridge discrete-continuum models, need to be reconsidered in the light of the findings of this paper.

\section{Summary and conclusion}

The granular micromechanics approach has been used to develop an enhanced continuum model of grain assemblies by the identification of grain (microscale) motions in terms of the macroscale displacement gradient, the fluctuations in displacement gradient as well as their second gradient. Thus, additional stress tensors conjugate to the strain measures as well as additional force measures that are conjugate to the grain-pair displacements are introduced. The expressions of stress tensors are found in terms of the corresponding force and geometric measures, which are different from those derived using the generalized virial theorem. Further, defining the macroscopic strain energy density as the volume average of grainpair energy functions, macroscopic stiffness tensors corresponding to the kinematic measures have been derived. The continuum stiffness tensors are obtained in terms of grain-pair stiffness coefficients and fabric parameters defining the geometry of grains and their contacts.

To identify the elastic constants of the enhanced continuum model, we perform numerical experiments on grain assemblies using discrete simulations subjected to relevant boundary conditions. The need for additional macroscale deformation measures for the continuum modeling of granular materials becomes evident in this identification process. The obtained elastic constants are then used to determine the microscale (or grain-pair) stiffness coefficients applicable to the continuum model. These grain-scale stiffness coefficients are found to be affected by the heterogeneity of microstructure, as shown by the results for regular grain assembly 
made increasingly heterogeneous by introducing vacancy-type defects. The grainpair stiffness coefficients are clearly different from the isolated grain-pair stiffness used in discrete simulation. These effective stiffness coefficients are unique for each material or granular assembly, since the intergranular mechanisms are affected not only by the two grains under consideration, but also by the grains in the neighborhood, and, by extension, the whole assembly. We also find that the stiffness coefficients corresponding to the average displacement gradient terms have the largest values but the coefficients corresponding to displacement gradient fluctuations are also significant and of a similar order.

Finally, we note that the micromorphic and second gradient terms are necessary for modeling some frequency-dependent wave transmission/reflection phenomena at material interfaces [Misra and Poorsolhjouy 2015b; Placidi et al. 2014; dell'Isola et al. 2012; Madeo et al. 2015]. The micromorphic behavior of granular materials indicates the possibility of realizing materials with alternate synthesis pathways which show specific wave propagation behaviors that can be used for vibration control as an alternative to piezoelectric materials [Maurini et al. 2004; 2006; Porfiri et al. 2005; Vidoli and dell'Isola 2001; Madeo et al. 2014; dell'Isola and Vidoli 1998; Greco et al. 2014] or for damage identification [Ferretti et al. 2014; Andreaus and Baragatti 2011; 2012]. Alternatively, such materials can be applied to help optimize control procedures [Andreaus et al. 2012; 2015a] or for optimal biomaterial design in bone mechanics [Andreaus et al. 2015b]. The identification process described herein can be used for extending the applicability of micromorphic models or their micropolar and second gradient simplifications to describe post-instability macroscale behavior, such as boundary and localization layers in microstructured media [Altenbach et al. 2010; Placidi 2015; Yang et al. 2011; Yang and Misra 2012].

\section{Appendix I. Expressions for elastic constants of 2D granular assemblies}

For defining grain-scale constitutive equations, a local coordinate system is introduced for each grain-pair interaction. This coordinate system is composed of a unit normal vector, $n_{i}$, in the direction of the branch vector joining the two grains' centroids and another unit vector, $s_{i}$, lying in the direction of the tangential plane, whose normal vector is $\boldsymbol{n}$. Cartesian components of the unit vectors are defined as

$$
n_{i}=\langle\cos \theta, \sin \theta\rangle, \quad s_{i}=\langle-\sin \theta, \cos \theta\rangle .
$$

Following the previous discussion about using an average value for the geometric tensors $l_{i}$ and $J_{i j}$ in any given orientation, and using the 2D density distribution function introduced in (17a), the summations in equations (14)-(16) are rewritten 
as integrals in the forms

and

$$
\begin{aligned}
& C_{i j k l}^{M}=\frac{1}{V} \sum_{\alpha} K_{i k}^{M} l_{l}^{\alpha} l_{j}^{\alpha}=l^{2} N_{p} \int_{\theta=0}^{2 \pi}\left(K_{i k}^{M} n_{j} n_{l}\right) \xi d \theta, \\
& C_{i j k l}^{m}=\frac{1}{V} \sum_{\alpha} K_{i k}^{m} l_{l}^{\alpha} l_{j}^{\alpha}=l^{2} N_{p} \int_{\theta=0}^{2 \pi}\left(K_{i k}^{m} n_{j} n_{l}\right) \xi d \theta,
\end{aligned}
$$

$$
\begin{aligned}
& A_{i j k l m n}^{g}=\frac{1}{V} \sum_{\alpha} K_{i l}^{g} J_{m n}^{\alpha} J_{j k}^{\alpha}=\frac{l^{4} N_{p}}{4} \int_{\theta=0}^{2 \pi}\left(K_{i l}^{g} n_{j} n_{k} n_{m} n_{n}\right) \xi d \theta, \\
& A_{i j k l m n}^{u}=\frac{1}{V} \sum_{\alpha} G_{p q}^{u} e_{m l q} e_{j i p} l_{k} l_{n}=l^{2} N_{p} \int_{\theta=0}^{2 \pi}\left(G_{p q}^{u} e_{l m q} e_{i j p} n_{k} n_{n}\right) \xi d \theta .
\end{aligned}
$$

Note that, since the method is applied here for only 2D modeling, the indices $i$, $j, k, l, m$, and $n$ take the value of either 1 or 2 . It should be noted here that in a 2D domain (with in-plane coordinate axes 1 and 2) the only possible rotation is the rotation about the axis normal to the plane under consideration, 3 axis, denoted as $\theta_{3}$, which gives rise to the moment component $m_{3}$. Now, in (31b), $e_{i j p}$ and $e_{l m q}$ denote permutation symbols, and since the indices $i, j, l$, and $m$ can take only values of 1 and 2 , the indices $p$ and $q$ should only take the value 3 . So the rotational stiffness tensor, $\boldsymbol{G}$, can have only one component, $G_{p q}=G_{33}=G$. Having this in mind and considering Equation (13) defining the general constitutive equations in intergranular scale, the moment-rotation constitutive equation in grain-scale can thus be written simply as

$$
m_{3}=G \theta_{3} .
$$

For the force-displacement constitutive equations in microscale, intergranular force and displacement vectors are decomposed in the local directions into two components, one normal and one tangential. Constitutive equations in the local level are then defined in this coordinate system as

$$
\left\{\begin{array}{l}
f_{n} \\
f_{s}
\end{array}\right\}=\left(\begin{array}{ll}
K_{n} & K_{n s} \\
K_{n s} & K_{s}
\end{array}\right)\left\{\begin{array}{l}
\delta_{n} \\
\delta_{s}
\end{array}\right\}
$$

where we have, for convenience, not shown the superscripts. As it is seen in (33), in the model presented here, the interaction between normal and tangential components of intergranular force and displacement vectors is included. In the previous versions of the method of granular micromechanics this coupling term was ignored, and the stiffness tensor was diagonal and was composed of one normal component, $K_{n}$, and one tangential component, $K_{s}$. It should be pointed out, however, that the stiffness tensor is still kept symmetric. The stiffness tensor used in (33) should then be rotated to the VE coordinate system to result in the stiffness 
tensor $K_{i j}$, which will be used in the grain-scale constitutive equation $f_{i}=K_{i j} \delta_{j}$ and also in equations (29), (30), and (31a), and which is given by

$$
K_{i j}=\left(\begin{array}{ll}
K_{11} & K_{12} \\
K_{21} & K_{22}
\end{array}\right)=\left(\begin{array}{ll}
n_{1} & s_{1} \\
n_{2} & s_{2}
\end{array}\right)\left(\begin{array}{cc}
K_{n} & K_{n s} \\
K_{n s} & K_{s}
\end{array}\right)\left(\begin{array}{ll}
n_{1} & n_{2} \\
s_{1} & s_{2}
\end{array}\right)
$$

For isotropic materials and in the linear elastic limit, constitutive equations and their corresponding stiffness tensors can be derived in closed form by performing the integrations presented in (29)-(31). The 2D form of the constitutive equations and corresponding stiffness tensors are thus derived as

$$
\begin{gathered}
\left\{\begin{array}{l}
\tau_{11} \\
\tau_{22} \\
\tau_{12}
\end{array}\right\}=\left(\begin{array}{ccc}
C_{11}^{M} & C_{12}^{M} & 0 \\
C_{12}^{M} & C_{11}^{M} & 0 \\
0 & 0 & C_{33}^{M}
\end{array}\right)\left\{\begin{array}{l}
\epsilon_{11} \\
\epsilon_{22} \\
\epsilon_{12}
\end{array}\right\}, \quad \text { where }\left\{\begin{array}{l}
C_{11}^{M}=\frac{1}{8} l^{2} N_{p}\left(3 k_{n}^{M}+k_{s}^{M}\right), \\
C_{33}^{M}=\frac{1}{8} l^{2} N_{p}\left(k_{n}^{M}+k_{s}^{M}\right), \\
C_{12}^{M}=\frac{1}{8} l^{2} N_{p}\left(k_{n}^{M}-k_{s}^{M}\right),
\end{array}\right. \\
\left\{\begin{array}{l}
\sigma_{11} \\
\sigma_{22} \\
\sigma_{12} \\
\sigma_{21}
\end{array}\right\}=\left(\begin{array}{llll}
C_{11}^{m} & C_{12}^{m} & C_{13}^{m} & C_{14}^{m} \\
C_{12}^{m} & C_{11}^{m} & C_{13}^{m} & C_{14}^{m} \\
C_{13}^{m} & C_{13}^{m} & C_{33}^{m} & C_{12}^{m} \\
C_{14}^{m} & C_{14}^{m} & C_{12}^{m} & C_{33}^{m}
\end{array}\right)\left\{\begin{array}{l}
\gamma_{11} \\
\gamma_{22} \\
\gamma_{12} \\
\gamma_{21}
\end{array}\right\}, \text { where }\left\{\begin{array}{l}
C_{11}^{m}=\frac{1}{8} l^{2} N_{p}\left(3 k_{n}^{m}+k_{s}^{m}\right), \\
C_{33}^{m}=\frac{1}{8} l^{2} N_{p}\left(k_{n}^{m}+3 k_{s}^{m}\right), \\
C_{12}^{m}=\frac{1}{8} l^{2} N_{p}\left(k_{n}^{m}-k_{s}^{m}\right), \\
C_{13}^{m}=-C_{14}^{m}=\frac{1}{4}-l^{2} N_{p} k_{n s}^{m},
\end{array}\right.
\end{gathered}
$$

and

$$
\left\{\begin{array}{l}
\mu_{111} \\
\mu_{112} \\
\mu_{121} \\
\mu_{122} \\
\mu_{211} \\
\mu_{212} \\
\mu_{221} \\
\mu_{222}
\end{array}\right\}=\left(\left[A_{i j k l m n}^{g}\right]_{8 \times 8}+\left[A_{i j k l m n}^{u}\right]_{8 \times 8}\right)\left\{\begin{array}{l}
\phi_{1,11} \\
\phi_{1,12} \\
\phi_{1,21} \\
\phi_{1,22} \\
\phi_{2,11} \\
\phi_{2,12} \\
\phi_{2,21} \\
\phi_{2,22}
\end{array}\right\}=\left[A_{i j k l m n}\right]_{8 \times 8}\left\{\begin{array}{l}
\phi_{1,11} \\
\phi_{1,12} \\
\phi_{1,21} \\
\phi_{1,22} \\
\phi_{2,11} \\
\phi_{2,12} \\
\phi_{2,21} \\
\phi_{2,22}
\end{array}\right\},
$$

where

$\left[A_{i j k l m n}^{g}\right]_{8 \times 8}=\frac{1}{16} l^{4} N_{p} \times$

$$
\left(\begin{array}{cccccccc}
5 k_{n}^{g}+k_{s}^{g} & -2 k_{n s}^{g} & -2 k_{n s}^{g} & k_{n}^{g}+k_{s}^{g} & 4 k_{n s}^{g} & k_{n}^{g}-k_{s}^{g} & k_{n}^{g}-k_{s}^{g} & 0 \\
-2 k_{n s}^{g} & k_{n}^{g}+k_{s}^{g} & k_{n}^{g}+k_{s}^{g} & -2 k_{n s}^{g} & k_{n}^{g}-k_{s}^{g} & 0 & 0 & k_{n}^{g}-k_{s}^{g} \\
-2 k_{n s}^{g} & k_{n}^{g}+k_{s}^{g} & k_{n}^{g}+k_{s}^{g} & -2 k_{n s}^{g} & k_{n}^{g}-k_{s}^{g} & 0 & 0 & k_{n}^{g}-k_{s}^{g} \\
k_{n}^{g}+k_{s}^{g} & -2 k_{n s}^{g} & -2 k_{n s}^{g} & k_{n}^{g}+5 k_{s}^{g} & 0 & k_{n}^{g}-k_{s}^{g} & k_{n}^{g}-k_{s}^{g} & -4 k_{n s}^{g} \\
4 k_{n s}^{g} & k_{n}^{g}-k_{s}^{g} & k_{n}^{g}-k_{s}^{g} & 0 & k_{n}^{g}+5 k_{s}^{g} & -2 k_{n s}^{g} & -2 k_{n s}^{g} & k_{n}^{g}+k_{s}^{g} \\
k_{n}^{g}-k_{s}^{g} & 0 & 0 & k_{n}^{g}-k_{s}^{g} & -2 k_{n s}^{g} & k_{n}^{g}+k_{s}^{g} & k_{n}^{g}+k_{s}^{g} & -2 k_{n s}^{g} \\
k_{n}^{g}-k_{s}^{g} & 0 & 0 & k_{n}^{g}-k_{s}^{g} & -2 k_{n s}^{g} & k_{n}^{g}+k_{s}^{g} & k_{n}^{g}+k_{s}^{g} & -2 k_{n s}^{g} \\
0 & k_{n}^{g}-k_{s}^{g} & k_{n}^{g}-k_{s}^{g} & -4 k_{n s}^{g} & k_{n}^{g}+k_{s}^{g} & -2 k_{n s}^{g} & -2 k_{n s}^{g} & 5 k_{n}^{g}+k_{s}^{g}
\end{array}\right)
$$


and

$$
\left[A_{i j k l m n}^{u}\right]_{8 \times 8}=\frac{l^{2} N_{p}}{2}\left(\begin{array}{cccccccc}
0 & 0 & 0 & 0 & 0 & 0 & 0 & 0 \\
0 & 0 & 0 & 0 & 0 & 0 & 0 & 0 \\
0 & 0 & G & 0 & -G & 0 & 0 & 0 \\
0 & 0 & 0 & G & 0 & -G & 0 & 0 \\
0 & 0 & -G & 0 & G & 0 & 0 & 0 \\
0 & 0 & 0 & -G & 0 & G & 0 & 0 \\
0 & 0 & 0 & 0 & 0 & 0 & 0 & 0 \\
0 & 0 & 0 & 0 & 0 & 0 & 0 & 0
\end{array}\right) .
$$

It is noteworthy that the two fourth-rank stiffness tensors, $C_{i j k l}^{M}$ and $C_{i j k l}^{m}$, are formally similar. The only difference between the two tensors is that their components are derived based on grain-pair stiffness coefficients corresponding to different intergranular phenomena. It should also be noted that since the average strain tensor is the symmetric part of the macroscale displacement gradient, the strain and its conjugate stress tensor have three components. Thus, the stiffness matrix linking the average strain tensor to the Cauchy stress, $C_{i j k l}^{M}$, is also symmetrized into a $3 \times 3$ matrix, while $C_{i j k l}^{m}$ is a $4 \times 4$ matrix.

\section{Appendix II. Variational principle and balance equations}

The variation of the internal deformation energy functional in terms of the macroscale measures is obtained as

$$
\begin{aligned}
\delta W=\int_{v} \delta W d V= & \int_{v}\left(\tau_{i j} \delta \epsilon_{i j}+\sigma_{i j} \delta \gamma_{i j}+\mu_{i j k} \delta \phi_{i, j k}\right) d V \\
=-\int_{v}\left(\tau_{i j}\right. & \left.+\sigma_{i j}\right)_{, j} \delta \bar{\phi}_{i} d V-\int_{v}\left(\mu_{i j k, k}+\sigma_{i j}\right) \delta \psi_{i j} d V \\
& \quad+\int_{s}\left(\tau_{i j}+\sigma_{i j}\right) n_{j} \delta \bar{\phi}_{i} d S+\int_{s} \mu_{i j k} n_{k} \delta \psi_{i j} d S,
\end{aligned}
$$

where we have used Gauss's divergence theorem and equations (4) and (6). The variation of external energy due to external actions on the system is written as

$$
\delta W^{\mathrm{ext}}=\int_{v} f_{i} \delta \bar{\phi}_{i} d V+\int_{v} \Phi_{i j} \delta \psi_{i j} d V+\int_{s} t_{i} \delta \bar{\phi}_{i} d S+\int_{s} T_{i j} \delta \psi_{i j} d S
$$

where $f_{i}$ is the noncontact volumic (body) force per unit volume, $t_{i}$ is the contact traction, defined as a surface force per unit area, $\Phi_{i j}$ is the noncontact volumic (body) double force per unit volume, and $T_{i j}$ is the contact double traction, defined 
as double force per unit area. Combining equations (38) and (39) results in

$$
\begin{aligned}
\int_{v}\left[f_{i}+\left(\tau_{i j}+\right.\right. & \left.\left.\sigma_{i j}\right)_{, j}\right] \delta \bar{\phi}_{i} d V+\int_{v}\left[\Phi_{i, j}+\left(\mu_{i j k, k}+\sigma_{i j}\right)\right] \delta \psi_{i j} d V \\
& +\int_{S}\left[t_{i}-\left(\tau_{i j}+\sigma_{i j}\right) n_{j}\right] \delta \bar{\phi}_{i} d S+\int_{S}\left[T_{i j}-\mu_{i j k} n_{k}\right] \delta \psi_{i j} d S=0,
\end{aligned}
$$

which leads to the following balance equations and traction boundary conditions:

$$
\left\{\begin{array} { r l } 
{ ( \tau _ { i j } + \sigma _ { i j } ) _ { , j } + f _ { i } } & { = 0 , } \\
{ \mu _ { i j k , i } + \sigma _ { j k } + \Phi _ { j k } } & { = 0 , }
\end{array} \quad \left\{\begin{array}{rl}
\left(\tau_{i j}+\sigma_{i j}\right) n_{j} & =t_{i}, \\
\mu_{i j k} n_{k} & =T_{j k} .
\end{array}\right.\right.
$$

\section{References}

[Alibert et al. 2003] J.-J. Alibert, P. Seppecher, and F. Dell'Isola, "Truss modular beams with deformation energy depending on higher displacement gradients", Math. Mech. Solids 8:1 (2003), $51-73$.

[Altenbach et al. 2010] H. Altenbach, V. A. Eremeyev, L. P. Lebedev, and L. A. Rendón, "Acceleration waves and ellipticity in thermoelastic micropolar media", Arch. Appl. Mech. 80:3 (2010), 217-227.

[Andreaus and Baragatti 2011] U. Andreaus and P. Baragatti, "Cracked beam identification by numerically analysing the nonlinear behaviour of the harmonically forced response", J. Sound Vib. 330:4 (2011), 721-742.

[Andreaus and Baragatti 2012] U. Andreaus and P. Baragatti, "Experimental damage detection of cracked beams by using nonlinear characteristics of forced response", Mech. Syst. Signal Process. 31 (2012), 382-404.

[Andreaus et al. 2012] U. Andreaus, M. Colloca, and D. Iacoviello, "An optimal control procedure for bone adaptation under mechanical stimulus", Control Eng. Practice 20:6 (2012), 575-583.

[Andreaus et al. 2015a] U. Andreaus, M. Colloca, and D. Iacoviello, "Optimal bone density distributions: numerical analysis of the osteocyte spatial influence in bone remodeling", Comput. Methods Programs Biomedicine 113:1 (09/08 2015), 80-91.

[Andreaus et al. 2015b] U. Andreaus, I. Giorgio, and A. Madeo, "Modeling of the interaction between bone tissue and resorbable biomaterial as linear elastic materials with voids", Z. Angew. Math. Phys. 66:1 (2015), 209-237.

[Auffray et al. 2015] N. Auffray, F. dell'Isola, V. A. Eremeyev, A. Madeo, and G. Rosi, "Analytical continuum mechanics á la Hamilton-Piola least action principle for second gradient continua and capillary fluids", Math. Mech. Solids 20:4 (2015), 375-417.

[Cauchy 1826-1830] A.-L. Cauchy, "Sur l'equilibre et le mouvement d'un systeme de points materiels sollicites par des forces d'attraction ou de repulsion mutuelle", pp. 188-212 in Excercises de mathemátiques, vol. 3, 1826-1830.

[Chang and Misra 1989] C. S. Chang and A. Misra, "Computer simulation and modelling of mechanical properties of particulates", Comput. Geotech. 7:4 (1989), 269-287.

[Chen and Lee 2003] Y. Chen and J. D. Lee, "Connecting molecular dynamics to micromorphic theory, (I): Instantaneous and averaged mechanical variables", Physica A 322 (2003), 359-376.

[Cosserat and Cosserat 1909] E. Cosserat and F. Cosserat, Théorie des corps deformables, A. Hermann, Paris, 1909. Translated as Theory of deformable bodies, Paris, A. Hermann, 1909. 
[dell'Isola and Vidoli 1998] F. dell'Isola and S. Vidoli, "Continuum modelling of piezoelectromechanical truss beams: an application to vibration damping", Arch. Appl. Mech. 68:1 (1998), 1-19.

[dell'Isola et al. 2012] F. dell'Isola, A. Madeo, and L. Placidi, "Linear plane wave propagation and normal transmission and reflection at discontinuity surfaces in second gradient 3D continua", $Z$. Angew. Math. Mech. 92:1 (2012), 52-71.

[dell'Isola et al. 2014] F. dell'Isola, U. Andreaus, and L. Placidi, "At the origins and in the vanguard of peridynamics, non-local and higher-gradient continuum mechanics: an underestimated and still topical contribution of Gabrio Piola", Math. Mech. Solids 20:8 (2014), 887-928.

[Eringen 1999] A. C. Eringen, Microcontinuum field theories, I: Foundations and solids, Springer, New York, 1999.

[Ferretti et al. 2014] M. Ferretti, A. Madeo, F. dell'Isola, and P. Boisse, "Modeling the onset of shear boundary layers in fibrous composite reinforcements by second-gradient theory", Z. Angew. Math. Phys. 65:3 (2014), 587-612.

[Ganghoffer 2010] J.-F. Ganghoffer, "On the generalized virial theorem and Eshelby tensors", Int. J. Solids Struct. 47:9 (2010), 1209-1220.

[Germain 1973] P. Germain, "The method of virtual power in continuum mechanics, 2: Microstructure”, SIAM J. Appl. Math. 25:3 (1973), 556-575.

[Greco et al. 2014] L. Greco, N. Impollonia, and M. Cuomo, "A procedure for the static analysis of cable structures following elastic catenary theory", Int. J. Solids Struct. 51:7-8 (2014), 1521-1533.

[Green and Rivlin 1964] A. E. Green and R. S. Rivlin, "Multipolar continuum mechanics", Arch. Ration. Mech. An. 17 (1964), 113-147.

[Hill 1952] R. Hill, “The elastic behaviour of a crystalline aggregate”, P. Phys. Soc. A 65:5 (1952), 349.

[Jenkins et al. 2005] J. Jenkins, D. Johnson, L. La Ragione, and H. Makse, "Fluctuations and the effective moduli of an isotropic, random aggregate of identical, frictionless spheres", J. Mech. Phys. Solids 53:1 (2005), 197-225.

[Madeo et al. 2014] A. Madeo, L. Placidi, and G. Rosi, "Towards the design of metamaterials with enhanced damage sensitivity: second gradient porous materials", Res. Nondestruct. Eval. 25:2 (2014), 99-124.

[Madeo et al. 2015] A. Madeo, P. Neff, I. D. Ghiba, L. Placidi, and G. Rosi, "Wave propagation in relaxed micromorphic continua: modeling metamaterials with frequency band-gaps", Continuum Mech. Therm. 27:4-5 (2015), 551-570.

[Maugin 2014] G. A. Maugin, "Some remarks on generalized continuum mechanics", Math. Mech. Sol. 20:3 (2014), 280-291.

[Maurini et al. 2004] C. Maurini, F. dell'Isola, and J. Pouget, "On models of layered piezoelectric beams for passive vibration control”, J. Phys. (France) IV 115 (2004), 307-316.

[Maurini et al. 2006] C. Maurini, J. Pouget, and F. dell'Isola, "Extension of the Euler-Bernoulli model of piezoelectric laminates to include 3D effects via a mixed approach", Comput. Struct. 84:22-23 (2006), 1438-1458. Composite Adaptive Structures: Modelling and Simulation.

[Mindlin 1964] R. D. Mindlin, "Micro-structure in linear elasticity", Arch. Ration. Mech. An. 16 (1964), 51-78.

[Misra 1998] A. Misra, "Particle kinematics in sheared rod assemblies", pp. 261-266 in Physics of dry granular media, edited by H. Herrmann et al., NATO ASI Series 350, Springer Netherlands, 1998. 
[Misra and Chang 1993] A. Misra and C. S. Chang, "Effective elastic moduli of heterogeneous granular solids", Int. J. Solids Struct. 30:18 (1993), 2547-2566.

[Misra and Jiang 1997] A. Misra and H. Jiang, "Measured kinematic fields in the biaxial shear of granular materials", Comput. Geotech. 20:3-4 (1997), 267-285.

[Misra and Poorsolhjouy 2015a] A. Misra and P. Poorsolhjouy, "Elastic behavior of 2D grain packing modeled as micromorphic media based upon granular micromechanics", J. Eng. Mech.-ASCE (2015). (in review).

[Misra and Poorsolhjouy 2015b] A. Misra and P. Poorsolhjouy, "Granular micromechanics based micromorphic model predicts frequency band gaps", Continuum Mech. Therm. (2015), 1-20.

[Misra and Poorsolhjouy 2015c] A. Misra and P. Poorsolhjouy, "Granular micromechanics model for damage and plasticity of cementitious materials based upon thermomechanics", Math. Mech. Solids (2015).

[Misra and Singh 2014] A. Misra and V. Singh, "Nonlinear granular micromechanics model for multi-axial rate-dependent behavior”, Int. J. Solids Struct. 51:13 (2014), 2272-2282.

[Misra and Singh 2015] A. Misra and V. Singh, "Thermomechanics-based nonlinear rate-dependent coupled damage-plasticity granular micromechanics model”, Continuum Mech. Therm. 27:4-5 (2015), 787-817.

[Navier 1827] C. L. Navier, "Sur les lois de l'equilibre et du mouvement des corps solides elastiques", pp. 375-393 in Memoire de l'academie royale de sciences, vol. 7, 1827.

[Peters and Walizer 2013] J. F. Peters and L. E. Walizer, "Patterned nonaffine motion in granular media”, J. Eng. Mech. (ASCE) 139:10 (2013), 1479-1490.

[Placidi 2015] L. Placidi, "A variational approach for a nonlinear 1-dimensional second gradient continuum damage model”, Continuum Mech. Therm. 27:4-5 (2015), 623-638.

[Placidi et al. 2014] L. Placidi, G. Rosi, I. Giorgio, and A. Madeo, "Reflection and transmission of plane waves at surfaces carrying material properties and embedded in second-gradient materials", Math. Mech. Solids 19:5 (2014), 555-578.

[Porfiri et al. 2005] M. Porfiri, F. dell'Isola, and E. Santini, "Modeling and design of passive electric networks interconnecting piezoelectric transducers for distributed vibration control", Int. J. Appl. Electrom. 21 (2005), 69-87.

[Seppecher et al. 2011] P. Seppecher, J.-J. Alibert, and F. dell'Isola, "Linear elastic trusses leading to continua with exotic mechanical interactions", J. Phys. Conf. Ser. 319:1 (2011), 012018.

[Toupin 1964] R. A. Toupin, "Theories of elasticity with couple-stress", Arch. Ration. Mech. An. 17 (1964), 85-112.

[Trentadue 2001] F. Trentadue, "A micromechanical model for a non-linear elastic granular material based on local equilibrium conditions”, Int. J. Solids Struct. 38:40-41 (2001), 7319-7342.

[Vidoli and dell'Isola 2001] S. Vidoli and F. dell'Isola, "Vibration control in plates by uniformly distributed PZT actuators interconnected via electric networks", Eur. J. Mech. A Solids 20:3 (2001), 435-456.

[Yang and Misra 2012] Y. Yang and A. Misra, "Micromechanics based second gradient continuum theory for shear band modeling in cohesive granular materials following damage elasticity", Int. J. Solids Struct. 49:18 (2012), 2500-2514.

[Yang et al. 2011] Y. Yang, W.-Y. Ching, and A. Misra, "Higher-order continuum theory applied to fracture simulation of nanoscale intergranular glassy film”, J. Nanomech. Micromech. 1:2 (2011), $60-71$. 
Received 23 May 2015. Revised 20 Jul 2015. Accepted 31 Aug 2015.

ANIL MisRA: amisra@ku.edu

Civil, Environmental and Architectural Engineering Department, University of Kansas, 1530 W. 15th Street, Learned Hall, Lawrence, KS 66045-7609, United States

PAYAM POORSOLHJOUY: payam_poorsolhjouy@ku.edu Civil, Environmental and Architectural Engineering Department, University of Kansas, 1530 W. 15th Street, Learned Hall, Lawrence, KS 66045-7609, United States 
EDITORIAL BOARD

ANTONIO CARCATERRA

ERIC A. CARLEN

FRANCESCO DELL'ISOLA

RAFFAELE ESPOSITO

ALBERT FANNJIANG

Gilles A. FranCFORT

Pierangelo MARCATI

JEAN-JACQUES MARIGO

PETER A. MARKOWICH

MARTIN OSTOJA-STARZEWSKI

PIERRE SEPPECHER

DAVID J. STEIGMANN

PAUl STEINMANN

PierRe M. SuQueT

MANAGING EDITORS

MICOL AMAR

CORRADO LATTANZIO

ANGELA MADEO

MARTIN OSTOJA-STARZEWSKI

ADVISORY BOARD

ADNAN AKAY

Holm AltenBaCH

MICOL AMAR

HARM ASKES

TEODOR ATANACKOVIĆ

VICTOR BERDICHEVSKY

GUY BOUCHITTÉ

ANDREA BRAIDES

ROBERTO CAMASSA

MAURO CARFORE

ERIC DARVE

FELIX DARVE

ANNA DE MASI

GianPiEtro DEL Piero

EMMANUELE Di BENEDETTO

BERNOLD FIEDLER

IRENE M. GAMBA

DAVID Y. GAO

SERGEY GAVRILYUK

TIMOTHY J. HEALEY

DOMINIQUE JEULIN

ROGER E. KHAYAT

CORRADO LATTANZIO

ROBERT P. LIPTON

ANGELO LUONGO

ANGELA MADEO

JUAN J. MANFREDI

CARLO MARCHIORO

GÉRARD A. MAUGIN

ROBERTO NATALINI PATRIZIO NEFF

ANDREY PIATNITSKI

ERRICO PRESUTTI

MARIO PULVIRENTI

LUCIO RUSSO

Miguel A. F. SANJUAN

PATRICK SElVADURAI

ALEXANDER P. SEYRANIAN

MIROSLAV ŠILHAVÝ

GUIDO SWEERS

ANTOINETTE TORDESILLAS

LEV TRUSKINOVSKY

JUAN J. L. VELÁZQUEZ VINCENZO VESPRI ANGELO VULPIANI msp.org/memocs

Università di Roma "La Sapienza", Italia

Rutgers University, USA

(CO-CHAIR) Università di Roma "La Sapienza", Italia

(TREASURER) Università dell'Aquila, Italia

University of California at Davis, USA

(CO-CHAIR) Université Paris-Nord, France

Università dell'Aquila, Italy

École Polytechnique, France

DAMTP Cambridge, UK, and University of Vienna, Austria

(CHAIR MANAGING EDITOR) Univ. of Illinois at Urbana-Champaign, USA

Université du Sud Toulon-Var, France

University of California at Berkeley, USA

Universität Erlangen-Nürnberg, Germany

LMA CNRS Marseille, France

Università di Roma "La Sapienza", Italia

Università dell'Aquila, Italy

Université de Lyon-INSA (Institut National des Sciences Appliquées), France

(CHAIR MANAGING EDITOR) Univ. of Illinois at Urbana-Champaign, USA

Carnegie Mellon University, USA, and Bilkent University, Turkey

Otto-von-Guericke-Universität Magdeburg, Germany

Università di Roma "La Sapienza", Italia

University of Sheffield, UK

University of Novi Sad, Serbia

Wayne State University, USA

Université du Sud Toulon-Var, France

Università di Roma Tor Vergata, Italia

University of North Carolina at Chapel Hill, USA

Università di Pavia, Italia

Stanford University, USA

Institut Polytechnique de Grenoble, France

Università dell'Aquila, Italia

Università di Ferrara and International Research Center MEMOCS, Italia

Vanderbilt University, USA

Freie Universität Berlin, Germany

University of Texas at Austin, USA

Federation University and Australian National University, Australia

Université Aix-Marseille, France

Cornell University, USA

École des Mines, France

University of Western Ontario, Canada

Università dell' Aquila, Italy

Louisiana State University, USA

Università dell'Aquila, Italia

Université de Lyon-INSA (Institut National des Sciences Appliquées), France University of Pittsburgh, USA

Università di Roma "La Sapienza”, Italia

Université Paris VI, France

Istituto per le Applicazioni del Calcolo "M. Picone", Italy

Universität Duisburg-Essen, Germany

Narvik University College, Norway, Russia

Università di Roma Tor Vergata, Italy

Università di Roma "La Sapienza”, Italia

Università di Roma “Tor Vergata”, Italia

Universidad Rey Juan Carlos, Madrid, Spain

McGill University, Canada

Moscow State Lomonosov University, Russia

Academy of Sciences of the Czech Republic

Universität zu Köln, Germany

University of Melbourne, Australia

École Polytechnique, France

Bonn University, Germany

Università di Firenze, Italia

Università di Roma La Sapienza, Italia

MEMOCS (ISSN 2325-3444 electronic, 2326-7186 printed) is a journal of the International Research Center for the Mathematics and Mechanics of Complex Systems at the Università dell'Aquila, Italy.

Cover image: "Tangle” by $\odot$ John Horigan; produced using the Context Free program (contextfreeart.org).

PUBLISHED BY

7 mathematical sciences publishers

nonprofit scientific publishing

http://msp.org/

(C) 2015 Mathematical Sciences Publishers 
Mathematics and Mechanics of Complex Systems vol. 3 no. 3

Stationary solutions of Keller-Segel-type crowd motion and 211 herding models: Multiplicity and dynamical stability

Jean Dolbeault, Gaspard Jankowiak and Peter

Markowich

Comprehensive description of deformation of solids as 243 wave dynamics

\section{Sanichiro Yoshida}

On the constitutive equations of viscoelastic micropolar 273 plates and shells of differential type

Holm Altenbach and Victor A. Eremeyev

Identification of higher-order elastic constants for grain 285 assemblies based upon granular micromechanics

Anil Misra and Payam Poorsolhjouy

MEMOCS is a journal of the International Research Center for the Mathematics and Mechanics of Complex Systems at the Università dell' Aquila, Italy.

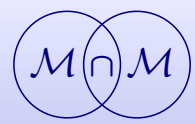

